\title{
Chiral Resolution of a New Agent against Memory Impairment Connected to Neurodegenerative Diseases ${ }^{\dagger}$
}

\author{
Valeria Cavalloro 1, Marta Rui 2, Giacomo Rossino ${ }^{2}$, Daniela Rossi ${ }^{2}$, Federica Rapetti ${ }^{3}$, \\ Chiara Brullo ${ }^{3}$, Olga Bruno ${ }^{3}$ and Simona Collina ${ }^{2, *}$ \\ 1 Earth and Environmental Sciences Department, University of Pavia, 27100 Pavia, Italy \\ 2 Department of Drug Sciences, University of Pavia, 27100 Pavia, Italy \\ 3 Department of Pharmacy, University of Genova, 27100 Pavia, Italy \\ * Correspondence: simona.collina@unipv.it \\ + Presented at the 2nd Molecules Medicinal Chemistry Symposium (MMCS): Facing Novel Challenges in \\ Drug Discovery, Barcelona, Spain, 15-17 May 2019.
}

Published: 7 August 2019

Keywords: Nerodegenerative diseases; PDE4 inhibitors; homochiral drugs; HPLC chiral resolution

Nowadays, the incidence of neurodegenerative diseases is increasing, and these disorders will become one of the main challenges for medicine and public health in future years. Particularly, memory loss characterizes many neurodegenerative pathologies, and it is often related to low levels of cyclic adenosine monophosphate (cAMP). During recent years, researchers have highlighted that inhibiting phosphodiesterase type 4 (PDE4)-mediated hydrolysis of cAMP could represent a powerful tool to contrast memory impairment. In 2017, we identified GEBR-32a as a new molecular entity able to full inhibit PDE4D, a specific PDE4 isoform [1]. GEBR-32a was able to restore memory in an Alzheimer's disease rodent model without causing emesis, which is the typical undesired effect of the major part of PDE4 inhibitors. Hence, it has been selected for preclinical studies. Since stereoselectivity is known to play a role for receptor ligands, to investigate the influence of chirality on the interaction of compounds with PDE4, the chiral resolution of GEBR-32a was performed. On the basis of our previous experience, the racemate GEBR-32a was resolved by chiral high-performance liquid chromatography (HPLC), this approach being an effective way for both the analytical and the preparative separations of chiral compounds. The analytical screening was performed on several chiral chromatographic columns, under different elution conditions, adopting isocratic modes. The optimized method was transferred into a semipreparative scale, and enantiomers were isolated within good enantiomeric excess. Configuration assignment studies are ongoing.

\section{Reference}

1. Ricciarelli, R.; Brullo, C.; Prickaerts, J.; Arancio, O.; Villa, C.; Rebosio, C.; Calcagno, E.; Balbi, M.; van Hagen, B.T.; Argyrousi, E.K.; et al. Memory-enhancing effects of GEBR-32a, a new PDE4D inhibitor holding promise for the treatment of Alzheimer's disease. Sci. Rep. 2017, 7, 46320, doi:10.1038/srep46320.

(C) 2019 by the authors. Submitted for possible open access publication under the terms and conditions of the Creative Commons Attribution (CC BY) license (http://creativecommons.org/licenses/by/4.0/). 